\title{
Notas sobre la legislación y el debate internacional en torno a los derechos territoriales indígenas
}

Girardo Zúilga N.'

\section{RESUMEN}

El contexto para entender las realidades indígenas hoy es planetario. Las Naciones Unidas se hace cargo de este tema porque concierne, en efecto, al planeta, o por lo menos al conjunto de Estados que se hacen representar en la ONU. Lo anterior no sólo quiere decir que las luchas territoriales indígenas estén ocurriendo en los diferentes países, sino que además dichas luchas han ido adquiriendo un carácter transnacional y también, por tanto, el debate y la producción de normas para regular los conflictos que les subyacen. El artículo reconstruye y analiza uno de los elementos fundamentales de este proceso, gracias al cual ha ido surgiendo a nivel internacional una doctrina acerca de los derechos territoriales indígenas: el debate llevado a cabo en el seno de las Naciones Unidas. Asimismo, se revisan las disposiciones atingentes al tema contenidas en el Convenio 169 de la OIT, y en la Declaración de Naciones Unidas sobre los Derechos de los Pueblos Indígenas del Mundo.

\footnotetext{
ABSTRACT

The context to understand the indigenous reality today is planetary. The United Nations are taken charge of this topic because it concerns to the planet indeed, or at least to the group of countries represented in the UNO. The previous doesn't only mean that the native territorial fights are happening in the different countries, but also that these fights are acquiring a transnational character and therefore the debate and production of norms to regularize such conflicts are acquiring that character too. The article reconstructs and analyzes one of the fundamental elements of this process thanks to which a doctrine of international level concerning the indigenous territorial rights has been appearing: the debate carried out in the center of the United Nations. Also, the related dispositions to the theme are revised in the Agreement 169 of the ILO, and in the
}

Declaration of United Nations on the Rights of the Indigenous People of the World.

\section{Introducción}

El presente artículo pretende pasar una rápida revista al debate llevado a cabo en el seno de las $\mathrm{Na}$ ciones Unidas en torno al tema de los derechos indígenas, y más particularmente, al de los derechos territoriales indígenas. Para ello se describe sumariamente la trayectoria de esta discusión, y más en detalle los dos instrumentos internacionales más relevantes en estas materias: el Convenio sobre Pueblos Indígenas y Tribales en Países Independientes $\mathrm{N}^{\circ} 169$ de la OIT, aprobado en el año 1989, y la Declaración de Naciones Unidas sobre los Derechos de los Pueblos Indígenas del Mundo, aún en discusión en la Comisión de Derechos Humanos de la ONU.

Parece de particular importancia, desde nuestra perspectiva, iniciar un debate en torno a estas materias, especialmente en circunstancias de que en la actualidad el Parlamento chileno se encuentra estudiando la aprobación del Convenio 169 de la OIT. Si éste es aprobado, corresponderá operacionalizar las numerosas normas que establece, lo cual puede abrir una interesante oportunidad para un diálogo - Pueblos Indígenas - Estado -, que hoy parece insubstancial.

\section{Reseña: del "Informe Cobo" a la "Declaración de Naciones Unidas sobre los Derechos de los Pue- blos Indígenas del Mundo"}

El debate llevado a cabo en el contexto de los foros

\footnotetext{
1 Jefe de la Unidad de Operaciones de la Dirección Ejecutiva de la "Red de Cooperación Institucional para Zonas de Pobreza Rural" - Red PRORURAL, Programa Intersectorial dependiente del Ministerio de Agricultura. Email: gzuniga@prorural.tie.cl
} 
internacionales, ha contribuido a la producción de una abundante reflexión y a la progresiva elaboración de una cierta doctrina internacional acerca de los derechos de los pueblos indígenas. En el curso de este debate, la discusión sobre los derechos territoriales indígenas ha ido adquiriendo una notable visibilidad y centralidad a la vez que ha ido llenándose de significado. Si bien es posible encontrar, como veremos, antecedentes tempranos de este debate, no es sino a partir de fines de la década de los '70 y comienzos de los ' 80 , que éste se intensifica.

Probablemente, el primer antecedente o referencia sobre "indígenas" en los instrumentos internacionales, se encuentre en el Pacto de la Sociedad de las Naciones, en virtud de cuyo artículo 22 los estados miembros tenían la tarea de "...contribuir al bienestar y al desarrollo de la 'población indígena' de las 'colonias y territorios' bajo su control" (Naciones Unidas 1996: 5). En el Pacto el uso del término "indígena" servía para distinguir a las potencias coloniales de los pueblos que vivían bajo su dominación, pero en la práctica la aplicación de estas disposiciones refería a "...territorios, delimitados por fronteras reconocidas a nivel internacional, más que a pueblos susceptibles de ser diferenciados por factores sociológicos, históricos o políticos" (Naciones Unidas 1996: 6). Más tarde, la Carta de Naciones Unidas aprobada en 1945, hará referencia en su artículo 73 a "...territorios cuyas poblaciones no se administran todavía completamente a si mismas", eliminándose de este modo la referencia a pueblos o poblaciones indígenas y haciéndose primar un criterio territorial por sobre cualquier consideración de carácter étnico o cultural. Sin embargo, el 15 de diciembre de 1960 la Asamblea General de Naciones Unidas, adoptó la Resolución 1541 (XV), en virtud de la cual los territorios a los que se refiere el Artículo 73 de la Carta son definidos como "territorios no autónomos". La definición de estos "territorios no autónomos" sintetiza en cierto modo las nociones anteriores, estableciendo que se trata de todo aquel territorio “...geográficamente separado y étnicamente o culturalmente distinto del país que lo administra" (Naciones Unidas 1996: 6). En cualquier caso, como es evidente, lo establecido en estas disposiciones tenía una ligazón directa con la situación en que se encontraban los países bajo control de las potencias coloniales, y en particular a su territorio y población.
Una de las primeras referencias específicas a la cuestión indígena hecha por un organismo del sistema de las Naciones Unidas, se encuentra en el libro "Las poblaciones aborígenes. Condiciones de vida y de trabajo de las poblaciones autóctonas de países independientes", publicado por la OIT en 1953. Según algunos autores (p. e. Ibarra 1992), el "Convenio relativo a la protección e integración de las poblaciones indígenas tribuales y semitribuales en los países independientes", conocido más ampliamente como Convenio 107, aprobado en 1957 por la OIT, habría tenido su origen en las reflexiones contenidas en este libro. En todo caso, la promulgación de este Convenio marca un hecho de la mayor importancia en el tratamiento de los asuntos indígenas a nivel internacional, ya que es el primer instrumento de derecho internacional en hacer referencia específica a los derechos de las poblaciones indígenas. Su aprobación marca el inicio de un proceso en el curso del cual los asuntos indígenas serían crecientemente incorporados y tomados en cuenta dentro del sistema de Naciones Unidas.

Algunos años más tarde, en 1966, la Asamblea General de Naciones Unidas aprobó el "Pacto Internacional de Derechos Económicos, Sociales y Culturales" y el "Pacto Internacional de Derechos Civiles y Políticos". Aún cuando no se hace referencia específica a los derechos de las poblaciones indígenas, su Artículo $1^{\circ}$ será utilizado, tiempo después, en forma recurrente por las organizaciones indígenas para demandar el reconocimiento del derecho de autodeterminación, para los "pueblos" indígenas:"

\footnotetext{
2 La demanda por "autodeterminación", crecientemente incorporada al discurso político de las organizaciones indígenas de todo el continente, se sostiene en la premisa argumentativa de que los indígenas considerados en forma colectiva, son "pueblos". En el derecho internacional los "pueblos" constituyen una categoría y un sujeto cuya calidad es atributiva de un cierto conjunto de derechos y sobre todo del derecho a la libredeterminación. Cuando el movimiento indígena reclama que se considere colectivamente a los indígenas como pueblos, se está reclamando, entonces, que se le atribuya el derecho a la libre-determinación. El movimiento indígena no intenta, por lo demás, disimular cuál es el objetivo político que se persigue a través del uso de esta noción: "En el interés por encontrar una denominación común, la mejor de ellas, quizá es la de pueblos indios, ya que la categoría de pueblo tiene un rango en los documentos del derecho internacional (ONU, OEA), donde se especifica que todos los pueblos tienen derecho a la autodeterminación". Esta cita corresponde a uno de los acuerdos tomados en el II Encuentro Continental Campaña 500 años de Resistencia Indígena (1991: 39), y es la ratificación de una resolución tomada por la Comisión Autodeterminación y Proyecto Político del I Encuentro Continental de Pueblos Indios, realizado en Julio de 1990 en Quito.
} 
"Todos los pueblos tienen el derecho a la libre determinación. En virtud de este derecho establecen libremente su condición política y proveen asimismo a su desarrollo económico, social y cultural" (en Ibarra 1992: 16).

El año 1965, la Subcomisión de Prevención de la Discriminación y Protección a las Minorías ${ }^{3}$ dependiente de la Comisión de Derechos Humanos de Naciones Unidas, resolvió llevar a cabo un estudio sobre la discriminación racial, el que fue finalizado en $1971 .{ }^{4}$ En el informe final el relator incluyó un capítulo titulado "Medidas adoptadas en relación con la protección a los pueblos indígenas". En su párrafo 1102 , el informe recomienda la realización de un estudio específico destinado a “ ....definir más a fondo los términos del problema ${ }^{5}$ y las medidas nacionales e internacionales que se requiere para resolverlo". La Subcomisión aceptó dicha recomendación, y por resolución 1589 del 21 de mayo de 1971 el Consejo Económico y Social de Naciones Unidas autorizó la realización del "Estudio del problema de la discriminación contra las poblaciones indígenas". 6

Los sucesivos informes preliminares de este estudio presentados en las sesiones de la Subcomisión, permitieron ir construyendo un discurso específico acerca de los derechos indígenas y orientar el tratamiento de los asuntos indígenas por parte de la Subcomisión. Los resultados parciales y final de este estudio, constituyeron una referencia primera para toda la reflexión que se desencadenaría al interior del sistema de Naciones Unidas a partir de los inicios de la década de los "70. El "Informe Cobo" presenta en forma extensa y exhaustiva un discurso argumentativo que describe y explica la situación de los pueblos indígenas del mundo en base a una variada casuística. ${ }^{7}$ Sobre esta base argumentativa reposan las proposiciones hechas por el relator especial, las que están contenidas en el capítulo "Conclusiones propuestas y recomendaciones". Allí el relator especial propone una definición de "Poblaciones indígenas":

"Por comunidades, poblaciones y naciones autóctonas, hay que entender aquellas que, ligadas

3 En adelante la "Subcomisión".

4 Ver Santa Cruz (1971). El sr. Santa Cruz fue el relator especial encargado de realizar el estudio.

5 Se refiere a la discriminación de que son objeto las poblaciones indígenas. por una continuidad histórica con las sociedades anteriores a la invasión y con las sociedades precoloniales que se desarrollaron sobre sus territorios, se consideran distintas de los otros elementos de las sociedades que dominan actualmente sobre sus territorios o parte de sus territorios. Corresponden en el presente a elementos no dominantes de la sociedad y están determinados a conservar, desarrollar y transmitir a las futuras generaciones los territorios de sus ancestros y su identidad étnica que constituyen la base de la continuidad de su existencia en tanto que pueblos, conforme a sus propios modelos culturales, a sus instituciones sociales y a sus sistemas jurídicos" (Naciones Unidas 1986b: párrafo 379). ${ }^{8}$

Como puede leerse, el "Informe Cobo" afirma que la existencia de los indígenas, en tanto que "pueblos", está ligada a la conservación, desarrollo y transmisión de su "territorio ancestral" y de su "identidad étnica". Sin "territorio" y sin "identidad" los indígenas no existirían en tanto que "pueblos". Entre los argumentos en que Cobo sustenta esta afirmación encontramos, en el mismo Informe Final del estudio:

"Es esencial conocer y comprender la relación particular, profundamente espiritual, que las poblaciones indigenas tienen con la tierra, elemento fundamental de su existencia y substrato de todas sus creencias, sus costumbres, sus tradiciones y su cultura" (Naciones Unidas 1986b: párrafo 196).

"Para los indígenas, la tierra no es sólo un objeto de posesión y de producción. La relación integral de la vida espiritual de las poblaciones indígenas con la Madre Tierra, con sus tierras tiene muchas y profundas incidencias. Además, la tierra no es una mercancía de la que uno pueda apropiarse, sino un elemento natural del que cada uno debe poder gozar libremente" (Naciones Unidas 1986b: párrafo 197).

En 1982 el Consejo Económico y Social de Naciones Unidas autorizó a la Subcomisión, a través de la resolución 1982/34, para establecer un "Grupo de

\footnotetext{
6 La Subcomisión encomendó la realización del estudio al sr. José Martínez Cobo. De ahí que el Informe Final del estudio se conozca y denomine corrientemente "Informe Cobo".

7 El Informe consta de 4 volúmenes y 4 adendums. Ver Naciones Unidas (1986a).

8 Traducido del francés.
} 
Trabajo sobre Poblaciones Indígenas". ${ }^{y}$ El Grupo de Trabajo

"consta de cinco miembros de la Subcomisión que desempeñan las funciones de expertos independientes a título personal. El Grupo de Trabajo celebra reuniones anuales con dos mandatos: la evolución de las normas relativas a los derechos de los pueblos indigenas y el examen de los acontecimientos relativos a la promoción y protección de los derechos humanos y las libertades fundamentales de los pueblos indigenas" (Daes 1996: 3).

Desde su creación, el Grupo de Trabajo ha animado y organizado en torno de sí parte importante del debate internacional sobre los asuntos indígenas en el seno de las Naciones Unidas. A sus sesiones anuales concurren cientos de representantes indígenas de todo el mundo, cuya participación se vio estimulada por la creación del "Fondo de contribuciones voluntarias para las poblaciones indígenas" aprobada por la Asamblea General de Naciones Unidas en 1985, cuyo fin es facilitar la asistencia de los indígenas a las sesiones del Grupo de Trabajo.

El primer período de sesiones del Grupo de Trabajo tuvo lugar en 1982, oportunidad en la cual se adoptó la resolución de utilizar como "definición operacional" de "poblaciones indígenas", aquella que el Informe Cobo incluía entre sus recomendaciones. A partir de ese año el Grupo de Trabajo se abocó a la preparación de un proyecto de Declaración sobre los derechos específicos de los pueblos indígenas. El Grupo de Trabajo terminó la redacción final del proyecto de "Declaración de las Naciones Unidas sobre los derechos de los pueblos indígenas" en su $11^{\circ}$ período de sesiones, en 1993 , y lo sometió a consideración de la Subcomisión, quien lo aprobó sin modificaciones, según consta en la resolución 1994/45. ${ }^{10}$ La Subcomisión también resolvió presentar el Proyecto de declaración a la Comisión de Derechos Humanos en su $51^{\circ}$ período de sesiones (febrero de 1995). En la oportunidad la Comisión de Derechos Humanos, según consta en la resolución 1995/32 del 3 de marzo de 1995, decidió establecer, con la aprobación del Consejo Económico y Social, un Grupo de Trabajo intersesional, al que se encomendó la tarea de elaborar un proyecto definitivo de declaración, trabajo aún en curso.

La reflexión llevada a cabo en el seno del Grupo de Trabajo, se nutrió de los resultados de una serie de seminarios y conferencias técnicas organizadas por Naciones Unidas, en las que se trataron temas específicos acerca de los cuales el Proyecto de Declaración debía definir normas y derechos. Entre otros podemos destacar:

a) Seminario de las Naciones Unidas sobre los efectos del racismo y la discriminación racial en las relaciones sociales y económicas entre poblaciones indígenas y estados realizado en Ginebra, Suiza, entre el 16 y el 20 de enero de 1989.

b) Reunión de expertos sobre la autonomía interna de las poblaciones indígenas, realizado en Nuuk (Groenlandia), entre el 24 y el 28 de septiembre de 1991.

c) Conferencia técnica de las Naciones Unidas sobre la experiencia práctica en el logro de un desarrollo autónomo sustentable y ecológicamente idóneo, realizado en Santiago entre el 18 y el 22 de mayo de 1992.

d) Seminario de expertos sobre experiencias prácticas con respecto a los derechos y reivindicaciones relativos a tierras indígenas, realizado en Whitehorse (Canadá), del 24 al 28 de marzo de 1996.

Paralelamente, el Grupo de Trabajo encomendó la realización de estudios y la confección de informes de modo de reunir antecedentes para la mejor definición de disposiciones concernientes a aspectos específicos de la declaración. Entre ellos tenemos:

a) Estudio sobre la protección del patrimonio de los pueblos indígenas (Naciones Unidas 1995).

b) La noción de Pueblo Indígena (Naciones Unidas 1996).

c) Los pueblos indígenas y su relación con la tierra (Naciones Unidas 1997).

d) Estudio sobre los tratados, convenios y otros acuerdos constructivos entre los Estados y las poblaciones indígenas.

9 En adelante el "Grupo de Trabajo".

10 Ver informe del $11^{\circ}$ período de sesiones del Grupo de Trabajo sobre Poblaciones Indígenas (Naciones Unidas 1993). 
La constitución del Grupo de Trabajo marcó el ingreso definitivo de los asuntos indígenas en el seno de las Naciones Unidas, proporcionando a la temática un estatus que nunca había tenido con anterioridad. En adelante, los asuntos indígenas estarán presentes cada vez con mayor visibilidad en los diferentes organismos de las Naciones Unidas y en cada uno de los eventos importantes de esta organización en la década de los '90."

Los argumentos avanzados en cada una de las instancias a que hemos hecho referencia aparecerán en forma reiterada en las resoluciones emitidas por diferentes conferencias y eventos internacionales. La colosal reflexión y debate inaugurado con la realización del Informe Cobo, tendrá en la aprobación del Convenio 169 de la OIT en 1989 y del "Proyecto de declaración de Naciones Unidas sobre los derechos de los pueblos indígenas del Mundo", los dos hitos de mayor importancia. Toda la producción argumentativa elaborada y considerada en el curso de las sesiones del Grupo de Trabajo, de la Subcomisión, de cada seminario, de cada declaración indígena, y por las $\mathrm{ONG}$, servirán de sustento y validarán el conjunto de normas de derecho internacional contenidas en estos instrumentos.

\section{El "Convenio sobre Pueblos Indígenas y Tribales en Países Independientes" $N^{\circ} 169$ de la Organi- zación Internacional del Trabajo}

El "Convenio sobre pueblos indígenas y tribales en países independientes" $12 \mathrm{~N}^{\circ} 169$ aprobado por la $76^{\circ}$ reunión de la Conferencia General de la Organización Internacional del Trabajo (OIT) realizada en Ginebra en junio de 1989, es el único instrumento

11 En 1990 la Asamblea General de Naciones Unidas resuelve declarar 1993 como el "Año Internacional de las Poblaciones Indígenas del Mundo" y posteriormente designa a Rigoberta Menchú como Embajadora de Buena Voluntad de las Naciones Unidas. En junio de 1992 se realiza en Río de Janeiro la "Conferencia de las Naciones Unidas sobre el Medio Ambiente y el Desarrollo", en la que se adopta el Capítulo $26 \mathrm{del}$ Programa 21 relativo a las poblaciones indígenas. En $1993 \mathrm{se}$ desarrolló en Viena la "Conferencia Mundial de Derechos Humanos", la que sirvió de escenario a lo que se ha dado en llamar "La Guerra de las S", cuando los delegados debatían la pertinencia de utilizar la denominación "Peoples" (pueblo) o "people" (poblaciones). La Conferencia además recomendó a la Asamblea General la proclamación de un Decenio Internacional de las Poblaciones Indígenas y la creación de un Foro permanente para las poblaciones indígenas en el sistema de las Naciones Unidas. La Asamblea General en su resolución 48/163 del 21 de diciembre de 1993, proclamó el Decenio Internacional, que comenzaría el 10 de diciembre de 1994. de derecho internacional hoy vigente concebido específicamente para salvaguardar de manera global los derechos de dichos pueblos. ${ }^{13}$ Los contenidos del preámbulo y del articulado del Convenio reflejan fielmente la evolución en la forma en que a nivel internacional se entienden, problematizan y abordan los asuntos indígenas. Mientras el Convenio 107 de 1957 perseguía la integración/asimilación de los indígenas a las respectivas sociedades y estados nacionales en los que se encontraban insertos, el nuevo Convenio pretende garantizar la existencia e integridad de los "pueblos indígenas". En el preámbulo del Convenio puede leerse a este respecto:

"Considerando que la evolución del derecho internacional desde 1957 y los cambios sobrevenidos en la situación de los pueblos indigenas y tribales en todas las regiones del mundo hacen aconsejable adoptar nuevas normas internacionales en la materia, a fin de eliminar la orientación hacia la asimilación de las normas anteriores;

Reconociendo las aspiraciones de esos pueblos a asumir el control de su propias instituciones y formas de vida y de su desarrollo económico y a mantener y fortalecer sus identidades, lenguas y religiones, dentro del marco de los Estados en que viven;" (OIT 1990: 33).

Aún cuando todo el articulado del Convenio está fundado en estas premisas, sus Artículos 2 y 4 ilustran fielmente la nueva orientación adoptada:

\section{"Artículo 2. 1) Los gobiernos deberán asumir la responsabilidad, de desarrollar con la participación}

12 En adelante "Convenio 169 " "Convenio".

13 Con anterioridad fueron elaborados otros instrumentos que hacían referencia a los "derechos de los pueblos". La Declaración Universal de los Derechos de los Pueblos, aprobada en Argel en 1976, consagra el derecho a la existencia, y a la autodeterminación política de los pueblos y a sus derechos económicos, y nuevas nociones como el derecho a la cultura, el medioambiente y a los recursos comunes. La Carta Africana de los Derechos Humanos, aprobada en Nairobi en junio de 1981 por la Cumbre de los Jefes de Estado y de Gobierno de la Organización de la Unidad Africana, constituye el primer instrumento intergubernamental que hace referencia a los derechos de los "pueblos"; reconoce los derechos clásicos y el derecho de los pueblos a la existencia, a la libre disposición de sus riquezas y de sus recursos naturales, al desarrollo, así como el derecho a la paz y a la seguridad y a un medioambiente satisfactorio y global propio a su desanollo (en Boisson 1982: 13-19). 
de los pueblos interesados, una acción coordinada y sistemática con miras a proteger los derechos de esos pueblos y a garantizar el respeto de su integridad" (OIT 1990: 35-36).

"Artículo 4.1) Deberán adoptarse las medidas especiales que se precisen para salvaguardar las personas, las instituciones, los bienes, el trabajo, las culturas y el medio ambiente de los pueblos interesados" (OIT 1990: 36-37).

Uno de los aspectos más controvertidos del nuevo Convenio, es la adopción de la denominación de "Pueblos Indígenas y tribales" definidas en el Artículo 1 del Convenio, en reemplazo de la denominación "poblaciones indígenas" y "poblaciones tribuales y semitribuales" utilizada en el Convenio 107.

\section{"Artículo 1. 1) El presente Convenio se aplica:}

a) a los pueblos tribales en países independientes, cuyas condiciones sociales, culturales y económicas les distingan de otros sectores de la colectividad nacional, y que estén regidos total o parcialmente por sus propias costumbres o tradiciones o por una legislación especial;

b) a los pueblos en países independientes, considerados indígenas por el hecho de descender de poblaciones que habitaban en el país o en una región geográfica a la que pertenece el país en la época de la conquista o la colonización o del establecimiento de las actuales fronteras estatales y que, cualquiera que sea su situación jurídica, conservan todas sus propias instituciones sociales, económicas. culturales y políticas, o parte de ellas" (OIT 1990: $35)$.

Los temores de numerosos Gobiernos de que tal definición pudiera alentar afanes secesionistas por parte de los beneficiarios del Convenio, llevó a que dentro del mismo Artículo 1 se estableciera que:

"La utilización del término 'pueblos' en este Convenio no deberá interpretarse en el sentido de que tenga implicación alguna en lo que atañe a los derechos que pueda conferirse a dicho término en el derecho internacional" (OIT 1990: 35).

Con esta cláusula de excepción, se hacía referencia específica a los Pactos Internacionales de Derechos
Humanos y al derecho de libre determinación que consagran (ver apartado anterior). La alta votación a favor de la aprobación del Convenio, pareciera demostrar que los temores de los estados, especialmente en lo concerniente a su integridad territorial. lograron ser exorcizados por esta cláusula. ${ }^{14} \mathrm{Sin}$ embargo, el escaso número de Estados que han ratificado el Convenio, demuestra que el fantasma de la desintegración territorial sigue presente en el imaginario de los Estados-nacionales. ${ }^{15}$

La Parte II (Tierras) del Convenio establece un conjunto de normas sobre las "tierras y territorios indígenas". El No 1 del Artículo 13 establece la premisa argumentativa en la reposan todos los derechos que consagra el Convenio en esta materia:

\section{"1. Al aplicar las disposiciones de esta parte del} Convenio, los gobiernos deberán respetar la importancia especial que para las culturas y valores espirituales de los pueblos interesados reviste su relación con las tierras o territorios, o con ambos, según los casos, que ocupan o utilizan de alguna otra manera, y en particular los aspectos colectivos de esa relación" (OIT 1990: 41).

Esta premisa es de uso recurrente en los instrumentos internacionales, y forma parte de una cierta doctrina sobre los derechos indígenas en la que se presenta como un principio prácticamente de aplicación universal. Un documento reciente de las Naciones Unidas conclúa:

“...la especificidad cultural de los pueblos indígenas, que está en el centro de la noción de 'indígena' en el derecho internacional contemporáneo. es indisociable del 'territorio'" (1996:13).

El No 2 del Artículo 13 define el campo de aplicación del término "tierra" y proporciona la primera y única definición operacional de "territorio indígena" existente en derecho internacional:

“2. La utilización del término 'tierras' en los artículos 15 y 16 deberá incluir el concepto de terri-

14328 votos a favor, uno en contra y 49 abstenciones

15 Según datos recientes (marzo de 2000) de la OIT, el Convenio 107 de 1957 sigue vigente en 20 países, mientras que sólo 13 han ratificado el Convenio 169. Estos últimos son: Bolivia. Colombia, Costa Rica, Dinamarca. Ecuador. Fiji, Guatemala. Honduras, México, Noruega, Países Bajos, Paraguay y Perú. En: http://www.ilo.org 
torios, lo que cubre la totalidad del hábitat de las regiones que los pueblos interesados ocupan o utilizan de alguna otra manera" (OIT 1990: 41). ${ }^{16}$

Según esta definición, el "territorio indígena" sería equivalente al 'habitat' ocupado o utilizado por los pueblos indígenas. Según se deduce del texto de los artículos 15 y 16 del Convenio, el significado atribuido a la noción de "territorio" tiene la ambición de designar un sistema que sería continente no sólo de "tierras" o "suelo" sino también de "recursos naturales" en general y de "recursos del subsuelo" en particular, a los que hace referencia el artículo 15. De modo, el "territorio indígena" correspondería a la totalidad de los recursos naturales, incluidos los del subsuelo, existentes en las regiones donde viven y que utilizan los pueblos indígenas. La importancia de esta definición radica en que junto con definir el "territorio indígena" como un sistema compuesto por recursos naturales, define a las "...regiones que los pueblos interesados ocupan o utilizan" como el espacio jurisdiccional en el que los derechos indígenas sobre dichos recursos tienen vigencia.

Veamos sintéticamente cuales son los derechos que el Convenio consagra en relación a las "tierras" y "territorios indígenas" así definidos ${ }^{17}$ :

- Derecho de los pueblos indígenas sobre la "... propiedad y de posesión sobre las tierras que tradicionalmente ocupan" (artículo 14, $\mathrm{N}^{\circ} 1$ ).

- Derecho a "...utilizar tierras que no estén exclusivamente ocupadas por ellos (los pueblos indígenas), pero a las que hayan tenido tradicionalmente acceso para sus actividades tradicionales y de subsistencia" (Artículo 14, $\mathrm{N}^{\circ}$ 1).

- Derecho a los recursos naturales existentes en sus tierras. "Estos derechos comprenden el derecho de esos pueblos a participar en la utilización, administración y conservación de dichos recursos" (Artículo 15, $\mathrm{N}^{\circ} 1$ ).

- Derecho de los pueblos indígenas a ser consultados por los Gobiernos “....antes de emprender o autorizar cualquier programa de prospección o explotación de los recursos existentes en sus tierras", cuando la propiedad de éstos pertenezca al Estado (Artículo 15, No 2 ).
- Derecho a "...participar siempre que sea posible en los beneficios que reporten..." las actividades extractivas de recursos naturales existentes en sus tierras (Artículo 15, $\mathrm{N}^{\mathrm{O}} 2$ ).

Derecho a “...percibir una indemnización equitativa por cualquier daño que puedan sufrir (los pueblos indígenas) como resultado de esas actividades" extractivas (Artículo 15, № 2 ).

- Derecho de los pueblos indígenas de aplicar sus propias “....modalidades de transmisión de los derechos sobre la tierra" entre sus miembros (Artículo 17).

- Derecho de los pueblos indígenas a ser beneficiados por los "programas agrarios nacionales" con "la asignación de tierras adicionales .... cuando las tierras de que dispongan sean insuficientes para garantizarles los elementos de una existencia normal o para hacer frente a su posible crecimiento numérico" (Artículo 19).

- Derecho de los pueblos indígenas a ser beneficiados por los "programas agrarios nacionales" con "...el otorgamiento de los medios necesarios para el desarrollo de las tierras que dichos pueblos ya poseen" (Artículo 19).

Aún cuando son pocos los Estados que han ratificado e incorporado a su legislación el Convenio, la vigencia de este instrumento internacional ha tenido grandes repercusiones. En primer lugar, sus disposiciones forman parte del ordenamiento legal de los Estados que lo han ratificado, sirviendo a la vez de referencia obligada a la definición y puesta en plaza de legislaciones especiales a través de las cuales, a menudo, se operacionalizan las políticas indígenas de los países.

En segundo lugar, numerosos organismos internacionales no gubernamentales, algunos de los cuales tienen el estatus de organismos consultivos de $\mathrm{Na}$ ciones Unidas, han incorporado las definiciones del Convenio dentro de sus propios ordenamientos internos, y los derechos que consagra dentro de sus programas de acción. Así por ejemplo, Amnistía

16 Destacado nuestro.

17 Ver OIT (1990: 41 a 44) 
Internacional (1992) para efectos de su trabajo sobre indígenas ha adoptado oficialmente la definición de "pueblo indígena" tal como se la define en el Convenio. La World Wide Fund For Nature (1996), también ha adoptado oficialmente la definición de "pueblos indígenas" del Convenio, y los derechos y medidas que éste consagra dentro de su "Declaración de principios".

En tercer lugar, los contenidos del Convenio (principios, derechos y medidas) constituyen una jurisprudencia obligada para el trabajo desarrollado por los organismos del sistema de Naciones Unidas, y en particular para la elaboración de la "Declaración de Naciones Unidas sobre los Derechos de los Pueblos Indígenas del Mundo". Así también, sus contenidos ilustran una doble dinámica en cuanto al discurso de las organizaciones indígenas: por una parte representa y contiene varias de las reivindicaciones que las organizaciones indígenas venían reclamando; y por otra parte, ha pasado a formar parte del discurso argumentativo y reivindicativo de las organizaciones indígenas de todo el mundo. ${ }^{18}$

En cuarto lugar, el Convenio sintetiza en su preámbulo y articulado un nuevo corpus conceptual a partir del cual se problematizan los asuntos indígenas en la actualidad: el uso de la noción "Pueblos indígenas"; el uso diferenciado de las nociones "tierra", "territorio" y "recursos naturales".

\section{El Proyecto de "Declaración de Naciones Uni- das sobre los Derechos de los Pueblos Indígenas del Mundo"}

El Grupo de Trabajo aprobó el texto del proyecto de "Declaración de las Naciones Unidas sobre los derechos de los pueblos indígenas" 19 en su $11^{\circ}$ período de sesiones (1993), y lo sometió a consideración de la Subcomisión quien lo aprobó sin modificaciones según consta en la resolución 1994/45. Desde entonces el Proyecto se encuentra en la Comisión de Derechos Humanos. Luego de la aprobación de la Declaración por parte de la Comisión, el proyecto debía seguir una corta tramitación, como adelantaba en 1994 un experto de la Subcomisión ${ }^{20}$, hasta ser aprobado por la Asamblea General de las Naciones Unidas, pero el hecho es que la Comisión aún no cuenta con un proyecto definitivo de declaración y las discusiones se prosiguen en el seno del Grupo de Trabajo Intersesional al que la Comisión encomendó esta tarea.
Hasta el momento de ser despachado a la Comisión de Derechos Humanos, el proyecto de Declaración estuvo radicado en dos organismos técnicos integrados por expertos independientes: El Grupo de Trabajo sobre Poblaciones Indígenas y la Subcomisión de Prevención de la Discriminación y Protección a las Minorías. En este contexto el trabajo de confección del proyecto se benefició de un tratamiento técnico-político, en el que participaron numerosos representantes indígenas y especialistas individuales e institucionales. Seguramente la tramitación del Proyecto en la Comisión estará menos sujeto a consideraciones de orden "técnico" y más a consideraciones de orden político, ya que la Comisión está integrada por representantes de Gobier$\operatorname{nos}(58)$.

En cualquier caso, aún cuando se trata todavía de un proyecto y sus disposiciones no entrarán en vigencia sino cuando sea aprobado por la Asamblea General, el texto de la Declaración constituye hoy una referencia obligada, tanto para las organizaciones indígenas de todo el mundo, como para los organismos de todo tipo que trabajan en torno a sus problemáticas. Su texto contiene un cierto número de premisas, definiciones y un extenso articulado (45 artículos) que abarca una amplia gama de aspectos y asuntos relacionados con los pueblos indígenas. Revisemos a continuación los contenidos pertinentes a nuestro trabajo.

En primer lugar, se establece que los "pueblos indígenas" son los beneficiarios y sujetos de los derechos que se consagran en la Declaración (preámbu-

18 En el caso de Chile, sólo tardíamente las organizaciones indígenas incorporan este elemento a su discurso políticoreivindicativo. En el año 1989, por ejemplo, AD-MAPU demandaba al estado chileno respetar los tratados internacionales, y en especial el Convenio 107 de la OIT (1989: 23), en circunstancias de que el Estado chileno nunca lo suscribió. Como hemos señalado, el año 1989 finalizó el proceso de revisión de este Convenio, aprobándose el Convenio 169. De todos modos, la demanda por la aprobación del Convenio 169 se fue instalando gradualmente en el discurso y en los procesos de negociación entre las organizaciones indígenas y el Gobierno chileno. Recordemos que una de las conclusiones a las que llegó recientemente la Mesa de Diálogo (GobicrnoOrganizaciones Indígenas-Empresarios) hace referencia a la ratificación del Convenio 169 por parte del Estado chileno.

19 Ver Informe del $11^{\circ}$ período de sesiones del Grupo de Trabajo sobre Poblaciones Indígenas (Naciones Unidas 1993). Ver igualmente, Naciones Unidas (1994).

20 Ver Bengoa (1994). 
lo y Artículo 1), y se afirma que éstos son iguales en derechos y dignidad a todos los demás pueblos (Naciones Unidas 1994: 1). Ello significa que los derechos definidos en la Declaración son de carácter colectivo y que su disfrute y pleno ejercicio corresponde a los "Pueblos indígenas". La Declaración define a estos pueblos como entidades colectivas definidas por sus características "...intrínsecos.... que derivan de sus estructuras políticas, económicas y sociales y de sus culturas, de sus tradiciones espirituales, de su historia y de su concepción de la vida" (Naciones Unidas 1994: 2). Al definirlos de este modo los hace sujetos de los derechos colectivos específicos consagrados en la Declaración; mientras que al establecer que son iguales en derechos a los demás pueblos los hace igualmente beneficiarios de los derechos que los instrumentos internacionales garantizan a los "pueblos". En virtud de esta premisa es que el Preámbulo de la Declaración afirma:

"Reconociendo que la Carta de las Naciones Unidas, el Pacto Internacional de Derechos Económicos, Sociales y Culturales y el Pacto Internacional de Derechos Civiles y Políticos afirman la importancia fundamental del derecho de todos los pueblos a la libre determinación, en virtud del cual éstos determinan libremente su condición política y persiguen libremente su desarrollo económico, social y cultural" (Naciones Unidas 1994: 2).

"Teniendo presente que nada de lo contenido en la presente Declaración podrá utilizarse para negar a ningún pueblo su derecho a la libre determinación" (Naciones Unidas 1994: 3).

En segundo lugar, la Declaración afirma el derecho de los pueblos indígenas "... a ser diferentes, a considerarse a sí mismos diferentes y a ser respetados como tales" (Naciones Unidas 1994: 1). Al efecto la Declaración reconoce "...la urgente necesidad de respetar y promover los derechos y características intrínsecos de los pueblos indígenas, especialmente los derechos a sus tierras, territorios y recursos..." (Naciones Unidas 1994: 2). De modo que la forma de garantizar el derecho a la diferencia, equivalente al derecho a existir en tanto entidades colectivas tal como se ha definido, reposaría en el respeto y promoción de los derechos de los pueblos indígenas, particularmente sobre sus tierras, territorios y recursos y, además, en la posibilidad de los pueblos indígenas de ejercer “....control (sobre) los aconte- cimientos que les afecten a ellos y a sus tierras, territorios y recursos..." (Naciones Unidas 1994: 2). El "control" al que se refiere el Preámbulo se operacionaliza evidentemente a través del ejercicio de la libre determinación, tal como lo dispone el Artículo 31.

En el plano de las definiciones pertinentes a la problemática a que este trabajo se consagra, cabe señalar algunas observaciones. Primeramente, el Convenio enuncia en forma separada las nociones de "tierras", "territorios" y "recursos". Según uno de los expertos independientes de la Subcomisión, "el concepto de tierra dice relación con la propiedad, con el uso y goce individual y comunitario de la tierra en su acepción física, material" (Bengoa 1994: 9), en tanto que el concepto de "Territorio"

“...comprende el medio ambiente total de las aguas, los mares costeros, los hielos marinos, la flora y la fauna y los demás recursos que tradicionalmente han poseído u ocupado o utilizado de otra forma" (Bengoa 1994: 9).

En efecto, el Artículo 26 de la Declaración señala textualmente en relación a la noción de "Territorio":

"Los pueblos indígenas tienen derecho a poseer, desarrollar, controlar y utilizar sus tierras y territorios, comprendido el medio ambiente total de las tierras, el aire, las aguas, los mares costeros, los hielos marinos, la flora y la fauna y los demás recursos que tradicionalmente han poseído u ocupado o utilizado de otra forma..." (Naciones Unidas 1994: 8).

El "territorio", según esta definición, correspondería a un sistema que contiene los recursos y elementos espaciales naturales de los que hacen uso y que poseen tradicionalmente los pueblos indígenas. Como podrá notarse, no se hace mención específica a los recursos del subsuelo. Esta definición se asemeja bastante a la noción de "hábitat" utilizada en el Convenio 169. Sin embargo, el Artículo 31 de la Declaración, que establece las formas específicas a través de las cuales debería operacionalizarse el derecho a la libre determinación de los pueblos indígenas, deja entrever con timidez un aspecto complementario de esta definición de sesgo ambientalista. Este artículo señala textualmente: 
"Los pueblos indígenas, como forma concreta de ejercer su derecho de libre determinación, tienen derecho a la autonomía o el autogobierno en cuestiones relacionadas con sus asuntos internos y locales, en particular la cultura, la religión, la educación, la información, los medios de comunicación, la salud, la vivienda, el empleo, el bienestar social, las actividades económicas, la gestión de tierras y recursos, el medio ambiente y el acceso de personas que no son miembros a su territorio, así como los medios de financiar estas funciones autónomas" (Naciones Unidas 1994: 10).

Puede observarse que la Declaración consagra el derecho de la libre determinación en cuestiones relacionadas a los asuntos locales, y entre ellos el "acceso de personas que no son miembros a su territorio". Ambos enunciados dejan entrever una noción de "territorio" como espacio jurisdiccional. Lo dispuesto en el Artículo 26, citado precedentemente, confirma esta interpretación. Al igual que el artículo 15 del Convenio 169, esta disposición define el "territorio indígena" como el "medio ambiente total" que "tradicionalmente han poseído u ocupado o utilizado de otra forma" los pueblos indígenas. Por tanto el "territorio indígena" es el espacio jurisdiccional donde tienen vigencia los derechos indígenas sobre los recursos que forman parte de dicho "medio ambiente total".

Los derechos de los pueblos indígenas que la Declaración consagra en relación a las "tierras", "territorios" y "recursos", pueden resumirse en lo siguientes: ${ }^{21}$

- Derecho a ser protegidos y obtener reparación de "todo acto que tenga por objeto o consecuencia enajenarles sus tierras, territorios o recursos;" (Artículo 7, letra b).

- Derecho a ser informados y otorgar o negar libremente su consentimiento al desplazamiento de que pudieran ser objeto de sus tierras o territorios; y a percibir una indemnización justa y equitativa en caso de acceder a ser desplazados (Artículo 10).

- Derecho "....a una protección y seguridad especiales en períodos de conflicto armado" (Artículo 11), y a permanecer en sus tierras y territorios y seguir disponiendo de sus medios de subsistencia (Artículo 11, letra c).
- Derecho a "...mantener y fortalecer su propia relación espiritual y material con sus tierras, territorios, aguas, mares costeros y otros recursos que tradicionalmente han poseído u ocupado o utilizado de otra forma..." (Artículo 25).

- Derecho a "...poseer, desarrollar, controlar y utilizar sus tierras y territorios, comprendido el medio ambiente total de las tierras, el aire, las aguas, los mares costeros, los hielos marinos, la flora y la fauna y los demás recursos que tradicionalmente han poseído u ocupado o utilizado de otra forma...".

- Derecho a la “....restitución de las tierras, los territorios y los recursos que tradicionalmente han poseído u ocupado o utilizado de otra forma y que les hayan sido confiscados, ocupados, utilizados o dañados sin su consentimiento ..." (Artículo 27).

- Derecho a la “...conservación, reconstitución y protección del medio ambiente total y de la capacidad productiva de sus tierras, territorios y recursos..." (Artículo 28).

- Derecho a "...determinar y elaborar las prioridades y estrategias para el desarrollo o la utilización de sus tierras, territorios y otros recursos..." (Artículo 30).

- Derecho a otorgar o negar su consentimiento a los Estados frente a "...cualquier proyecto que afecte a sus tierras, territorios y otros recursos, particularmente en relación con el desarrollo, la utilización o la explotación de recursos minerales, hídricos o de otro tipo".

- Derecho a la autonomía o el autogobiemo en cuestiones relacionadas con, entre otras, "la gestión de tierras y recursos, el medio ambiente y el acceso de personas que no son miembros a su territorio..." (Artículo 31).

\section{Comentario final}

A partir de la década de los ' 70 es posible constatar el inicio de un proceso de transformación radical de las condiciones con arreglo a las cuales tienen lugar

21 Ver Naciones Unidas (1994). 
las relaciones entre las sociedades indígenas y los Estados Nacionales.

¿Cuáles son los factores que permitirían entender este proceso de transformación? Bien podría afirmarse que éste tiene su origen en los cambios políticos, sociales, económicos, demográficos y ambientales que han afectado o están afectando a los países de la región, por no hablar del mundo entero, y que han tenido como consecuencia la crisis y pérdida de vigencia de los sistemas normativos y categoriales que regularon en forma más o menos estable (gracias en buena parte a la acción autoritaria de los Estados) las relaciones sociales interétnicas hasta más o menos la década de los ' 80 .

Pero todas estas transformaciones, que aún no precipitan en sistemas estables, tienen lugar en un contexto aún más amplio, que concierne, por decirlo de un modo sencillo pero rotundo, al mundo entero. Aún a riesgo de simplificar en exceso el análisis, llamaremos a este contexto mundialización o globalización, que, en lo que nos interesa, está implicando una creciente complejización e intensificación de las relaciones internacionales o transfronterizas y, a la vez, una complejización e intensificación, pero sobre todo una profunda recomposición, de las relaciones intra-fronterizas, al interior de los Estados-nación. Aún más, la difusión y circulación masiva de bienes, ideas y personas, a través del uso de tecnologías cada vez más eficaces e, igualmente, más accesibles a los actores, también ha hecho posible que éstas --las relaciones internacionales y las intranacionales e interétnicas-- tengan lugar en niveles e intensidad y con interlocutores que antes no estaban articulados en campo o sector alguno de interacción.

Y las sociedades indígenas no han estado al margen de estos procesos. La cuestión étnica, o más específicamente los conflictos entre las sociedades indígenas y los Estados nacionales, no es un asunto nuevo en América Latina. Sin embargo, en los últimos treinta años empieza a adquirir rasgos novedosos, ganando visibilidad casi paralelamente a la emergencia de conflictos étnicos en otros sectores del globo. Como señala Bonfil Batalla,

"En muchos países del área hubo en esos años (los años que siguieron a la gran depresión y en especial a la segunda guerra mundial) una serie de procesos económicos y sociales que parecían anunciar que las identidades étnicas indias, tércamente vivas tras más de cuatro siglos de dominación, iban finalmente a disolverse y sus portadores asumirian de manera definitiva la identidad nacional correspondiente" (1988: 81).

En efecto, las poblaciones indígenas sufrieron todas las consecuencias de los procesos modernizadores puestos en plaza, que a través de la asalarización, la urbanización, la extensión de la ciudadanía política y la educación, permitieron la integración de enormes contingentes de población --que no solo indígenas-- que fueron desarraigados de sus formas de vida tradicionales. Por ello es que hoy, por ejemplo, se constata que una gran cantidad de población indígena reside en medios urbanos. Con todo, las sociedades indígenas, incluida aquella parte que protagonizó procesos migratorios y se instaló en las ciudades, fueron capaces de reintegrarse adaptativamente generando nuevas formas de organización social, refugiándose en algunos casos en sus comunidades rurales, reivindicando su pertenencia étnica en las grandes ciudades, constituyendo movimientos políticos de gran envergadura, generando alianzas políticas más allá de las fronteras nacionales, y elaborando y haciendo circular un discurso político transnacional, que igualmente se ha beneficiado de un cada vez más intenso intercambio político entre organizaciones indígenas de las más diversas realidades, que hacia fines de los años ' 80 presenta rasgos del todo novedosos: la demanda por "tierras" deja paso a una reivindicación por "territorios", la demanda por mayor "participación" da lugar a una demanda por "autonomía", por "autodeterminación", y las poblaciones indígenas que durante un largo período --durante el reinado de las dictaduras y la etapa inicial del despliegue del modelo neoliberal-- se habían refugiado en sus "comunidades", reaparecen en la escena política reivindicando les sea reconocido el estatus de "pueblos".

Hay en la base de todo este proceso una profunda puesta en cuestión del principio territorial que daba al orden mundial una cierta fisonomía. Bertrand Badie lo dice del siguiente modo:

"nuestra escena mundial se mundializa precisamente porque ella es irrigada por un conjunto de relaciones que obtienen su fuerza, que sacan provecho de su ignorancia del territorio Estado-nacional" (Badie 1997: 114). 
Ello se expresa en que

"las redes por intermedio de las cuales se lleva a cabo lo esencial de la vida económica, de la vida cultural, de comunicación, tienen lugar fuera de todo anclage territorial, fuera de toda codificación territorial, fuera de toda institucionalización territorial" (Badie 1997: 116).

En este contexto, la soberanía, y por tanto la seguridad, no se asegura tanto a través del control del territorio, sino a través de la participación en una comunidad internacional que intenta regular los flujos de todo tipo a través de los cuales se realiza la economía, la política, la cultura, la seguridad, etc. Sin embargo, según señala Gottmann (1973), sin territorio no se puede acceder a esta comunidad internacional: "El territorio sirve como una union card", dice Gottmann, "sin esta carta de acceso no se puede participar en la sociedad mundial". Esto pone de relieve la capacidad del territorio de constituirse en una función reguladora de las relaciones entre dos entidades sociopolíticas. La situación que afecta a las sociedades indígenas al interior de los Estados parece no ser tan distinta de la situación que afecta a los Estados en el contexto de la comunidad internacional. En algún sentido puede afirmarse que el "territorio indígena" es a los pueblos indígenas en el contexto de las relaciones con el Estado-nación, lo que el "territorio nacional" es a los Estados en el contexto de las relaciones interestáticas: la posesión de un territorio proporciona a los Estados-nación el atributo que requieren para existir frente a sus pares; mientras que la posesión de un territorio proporciona a las sociedades indígenas el atributo que requieren para constituirse en entidades sociopolíticas frente a los estados.

En resumen, la situación internacional presenta hoy una dinámica aparentemente contradictoria: de una parte la mundialización y los procesos que conlleva, que soslaya las fronteras, los territorios nacionales, frente a lo cual la comunidad internacional se dota crecientemente de mecanismos, instancias y normas de regulación en los más diversos ámbitos, aún, en todo caso, ineficaces e inequitativas; y de otra, los Estados (sobretodo los débiles) procuran revitalizar el principio territorial, porque es el territorio el que les proporciona esa union card, de que habla Gottmann, para participar de dicha comunidad mundial.
Pero, llendo un poco más allá en el análisis, el territorio, esta vez considerado como categoría objetiva, como espacio, tiene también que ver con la forma específica en que los Estados-nación participan de los procesos de generación, circulación y realización de la riqueza en el mundo, y esto tiene fuertes consecuencias en las formas como se da tratamiento al tema de los derechos indígenas al interior de sus fronteras.

Desde nuestra perspectiva, en los países cuyos modos de producción predominantes están organizados en torno a la producción y venta de conocimiento al interior de las fronteras y la realización de abundante plusvalía fuera de sus fronteras, a través de la venta de dicho conocimiento, de la deslocalización de su industria liviana (de productos manufacturados de consumo masivo) a países que ofrecen ventajas comparativas (menores costos de producción, por el costo del salario, beneficios tributarios, etc.) y la extracción de recursos naturales; pero que a la vez dominan el mercado de las relaciones internacionales, es posible implementar con éxito, como se está haciendo por ejemplo en Canadá o se hizo en el pasado en Dinamarca con Groenlandia, soluciones que han significado para los pueblos indígenas grandes concesiones territoriales y grados crecientes y sustantivos de autonomía para gestionar sus asuntos. Sin embargo, en países que, como Chile, parecen impedidos de renunciar a la explotación de sus recursos naturales y, por tanto, al control sobre la ocupación y uso del espacio, del territorio, y cuya inserción y capacidad de negociación en el concierto de esta comunidad internacional que se mundializa, es a toda evidencia reducida, tales soluciones parecen inviables porque amenazan aún más, desde dentro, aquella soberanía y territorialidad que desde ya está siendo evidentemente amenazada y puesta en cuestión desde fuera.

De este modo, la elaboración de normas internacionales no sólo tiene por objeto el reconocer e intentar garantizar un conjunto de derechos a las sociedades indígenas, porque se esté reconociendo la legitimidad y justicia de sus demandas. Tiene igualmente por objeto proporcionar un nuevo marco regulatorio y categorial a unas relaciones que han cambiado desde lo profundo, como hemos intentado mostrar en este comentario final, y que amenazan con constituirse en la principal causa de conflictos de gran envergadura a nivel planetario. Es posible que las normas internacionales constituyan 
los códigos que permitan o contribuyan a tratar esa "esquizofrenia estructural" de que nos habla Castell (1998), cuando caracteriza la situación de transformación y crisis que afecta al conjunto de las sociedades.

"En esta situación de esquizofrenia estructural - dice Castell-entre función y significación, los modelos de comunicación social sufren una tensión creciente. Y cuando la comunicación se rompe, cuando ella cesa de existir, incluso bajo la forma del conflicto (como en las luchas sociales o la oposición politica), individuos y grupos sociales toman sus distancias y perciben al otro como un extranjero, luego como una amenaza" (1998: 24).

El debate internacional (aún en curso) en torno a los derechos indígenas, y el subsecuente discurso que las organizaciones indígenas han elaborado y hecho circular para participar en él, pone de relieve el tema del territorio y los derechos territoriales (en cuanto su realización tiene lugar en el territorio), pero aún se está lejos de llegar a códigos compartidos que sean asumidos por los actores como el nuevo marco regulatorio que permitirá dar una nueva, y probablemente más justa, estabilidad a las relaciones entre los Estados-nación y las sociedades indígenas.

\section{Agradecimientos}

Agradezco los comentarios hechos a este artículo por Jorge Vergara y Hans Gundermann.

\section{BIBLIOGRAFIA}

AMNISTIA INTERNACIONAL, 1992. Los pueblos indigenas de América siguen sufriendo, Editorial Amnistía Internacional, Madrid

BADIE, B., 1997. La fin des territoires westphaliens, en, Géographie et cultures, L'Harmattan, № 20 hiver 1996, número especial territorio, pp. 113-118.

BENGOA J. 1994. Declaración de las Naciones Unidas sobre los Derechos de las Poblaciones Indígenas, Instituto de Estudios Indígenas-Universidad de la Frontera, Temuco, (Serie Documentos).

BOISSON, 1982. Introducción: los derechos de los Pueblos. En América Latina: Etnodesarrollo y Etnocidio, pp. 13-19. Ediciones FLACSO, San José de Costa Rica, (Colección 25 aniversario).

BONFIL BATALLA, G. 1988. Identidad étnica y movimientos indios en América Latina. En La cara india, la cruz del ' 92. Identidad étnica y movimientos indios. J. Contreras (Comp.) pp. 81-94. Editorial Revolución, Madrid.

CASTELL, M., 1998. La société en reseaux. Paris, Fayard.

DAES, E.-I., 1996. Panorámica concisa de las actividades del sistema de las Naciones Unidas relativas a los pueblos indígenas. En, Naciones Unidas, Informe del Seminario de Expertos sobre las experiencias prácticas con respecto a los derechos y reivindicaciones relativos a tierras indígenas, Dcto. N E/CN.4/Sub.2/AC.4/ 1996/6/ Add. 1, pp. 2-12.

GOTTMAN, J., 1973. The significance of territory, Charlettesville. The University Press of Virginia.
IBARRA, M., 1992. Practicas tradicionales sobre el desarrollo autónomo sostenible y ecológicanente idóneo de las poblaciones indígenas, Dcto. $\mathrm{N}^{\circ} \mathrm{H} / \mathrm{STGO} / 1992 / \mathrm{SEM} / \mathrm{BP}$.2. Santiago de Chile.

NACIONES UNIDAS, 1985. Dcto.E/CN.4/Sub.2/AC.4/1985/ WP.4.

----1986a. Estudio del problema de la discriminación contra las poblaciones indigenas, Dcto. $\mathrm{N}^{\circ} \mathrm{E} / \mathrm{CN} .4 / \mathrm{Sub} .2 / 1986 / \mathrm{A} 7 /$ Add. I a 4.

---- 1986b. Dcto. E/CN.4/Sub.2/1986/7/ Add.4; Publication des Nations Unies, $\mathrm{N}^{\circ}$ de vente F.86.XIV.3.

---- 1993. Deto. E/CN.4/Sub.2/1993/29, Anexo I.

---- 1994. Examen Técnico del proyecto de declaración de las Naciones Unidas sobre los derechos de las poblaciones indigenas-Adición Proyecto de Declaración Convenido por los miembros del Grupo de Trabajo en su $11^{\circ}$ período de sesiones, Dcto. E/CN.4/Sub.2/1994/2/Add.1.

---- 1995. Dcto. No E/CN.4/Sub.2/1995/ 26

-1.-- 1996. Document de travail du Président-Rapporteur. Mune Erica-Irene Daes, sur la notion de Pelple autochtone, Dcto. $N^{\circ}$ E/CN.4/Sub.2/ AC.4/1996/2, Genève.

-----1997. Document de travail préliminaire établie par Mme Erica-Irene Daes, Rapporteur spécial, Dcto. No E/CN.4/ Sub.2/1997/17.

OFICINA INTERNACIONAL DEL TRABAJO, 1989. Convenio 107 (www.oit.org) 
---- 1990. Convenio No 169 Sobre Pueblos Indígenas y Tribales en Países Independientes, en, COMISION ESPECIAL DE PUEBLOS INDIGENAS, Convenio $N^{o} 169$ sobre pueblos Indigenas y tribales en países independientes 1989, Editado por Comisión Especial de Pueblos Indígenas, Santiago de Chile, pp. 33-55, (Serie Documentos).

1991. Segundo Encuentro Continenal Campaña 500 años de Resistencia Indígena y Popular, Documentos de Conclusiones, Quetzaltenango, 7-12 de octubre, 1991.
SANTA CRUZ, H., 1971. La discriminación racial. Naciones Unidas, Nueva York, ( $\mathrm{N}^{\circ}$ de venta S.71.XIV.2)

WORLD WIDE FUND FOR NATURE, 1996. Los pueblos Indígenas y la Conservación: Declaración de Principios del WWF, WWF Internacional, Gland (Suiza), (WWF Internacional - Posición Oficial). 